\title{
Case Presentations and Evolution in Concepts of Immediate Loading in Edentulous Mandible
}

Keywords: Immediate loading; Splinting of implants; Paralleling guide; Optimal position; Angulation of implants

\begin{abstract}
The purpose of thiscase seriesisto demonstrate a n innova tive paralleling device and a step by step surgical and prosthetic approach for achieving buccolingual and mesiodistal parallelism of immediately loaded implants. Clinical outcomes including success rates, indications, advantages complic ations and limitations of this technique in cases using an Immediate Loading (IL) protocol will be disc ussed. Thirteen consecutive cases from the database that were treated with an IL protocol utilizing a paralleling device were included in this study. The treatment sites included the interforamina area in 8 totally edentulous and 5 partially edentulous mandibles. In this case series the average survival rate was $98.4 \%$ with a loading period of $6-28$ months. There was one implant failure, one provisional restoration fractured and two cases with cement wash out during the follow-up period. Results of this case series demonstrated that implant placement in optimal position and angulation using the suggested IL protocol and paralleling device producespredictable results. It is a lso simpler, fasterand more cost effective than the screw retained protocol.
\end{abstract}

\section{Introduction}

Increasing patient's expectations and the need for a fixed dentition during implant treatment led to the development of implant immediate loading procedures with provisional fixed prostheses [14]. The benefits of immediate implant loading include the restoration of esthetics, improving self-esteem, shortening treatment times, eliminating issues arising from the use of removable prostheses and a more rapid post-operative functional recovery [5]. Subsequently, there is gradual reincorporation of a normal diet with a more balanced nutrition.

In 1985, Schroeder introduced a single stage approach with TPS implants in which the coronal part of the one piece solid implant was placed slightly supramucosally, led to predictable clinical results [6]. Ericsson et al were among the first to show that turned implants could osseointegrate equally well with a one-stage approach in the interforaminal area of the mandible without jeopardizing bone remodeling [7]. Even after 5 years in function, implant survival and bone remodelling were comparable in the anterior mandible [8].

Several investigators thereafter, documented high success rates with the use of immediate and early loaded implants supporting full-arch fixed restorations in the mandible [9-13]. Immediate loading results for such restorations are dependent on the number of implants, the type of prosthesis, the presence or absence of splinting, the occlusal scheme, and the jaw being restored [14]. The use of immediate loading of splinted implants to restore edentulous arches has been documented. Tarnow et al. published their experiences with

\section{Journal of} Oral Biology

\author{
Ki Bum Kim*, Bhupesh Mandali, Wendy CW Wang \\ and Zahra Bagheri
}

Department of Periodontology and Implant Dentistry, New York University, USA

\section{*Address for Correspondence}

Ki Bum Kim, Department of Periodontology and Implant Dentistry, New York University, Clinic 5W, 345 E 24th St, New York, NY 10010, USA, Tel: +1-212-992-7040; E-mail: kbk211@nyu.edu

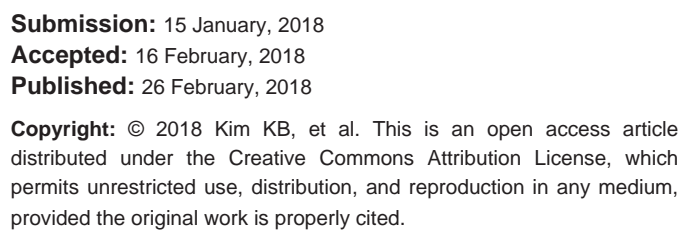

the immediate loading of edentulous arches in 10 patients [3]. The group placed 107 implants, 50 of which were immediately loaded. Two failures were recorded in the mandible of the experimental group. The authors attributed the failures to removal of the provisional prostheses for evaluation of implant healing. Tarnow et al proposed that cross-arch stabilization should be attempted in edentulous arches by splinting the implants by using a single provisional restoration. In addition, they also recommended leaving the provisional intra-orally at least two months post placement.

Despite following these guidelines, immediate loading still remains a technique sensitive procedure which requires precise implant placement and fabrication of an accurate provisional restoration for loading the implants. This is especially challenging in the mandibular anterior region where the arch's curvature is greatest and the alveolar crest is often uneven. Therefore various techniques have been introduced to make this procedure less technique sensitive, less time consuming, more accurate and more predictable. These included computer guided surgery, laboratory processed provisional, use of an Omnivac shell to fabricate cement retained provisional, use of angled abutments and use of temporary abutments to adjust the angulation during provisionalization $[3,15]$. These techniques are either expensive or time consuming yet not predictable. The latter of these techniques would not be necessary if implants were placed in optimal position and angulation with proper interimplant distance.

The purpose of this case series is to introduce an innovative technique that is cost effective, fast and predictable. A paralleling device and step by step guide for surgical and prosthetic rehabilitation approach with cement retained restoration for achieving buccolingual and mesiodistal parallelism of immediately loaded implants is demonstrated. Clinical outcomes including success rates, indications, advantages, complications, and limitations of this technique in cases using an immediate loading protocol will be discussed.

\section{Materials and Methods}

Clinical data in this study was obtained from the Implant Database (ID). This data was extracted as de-identified information from the routine treatment of patients at the Ashman Department of 
Citation: Kim KB, Mandali B, Wang WCW, Bagheri Z. Case Presentations and Evolution in Concepts of Immediate Loading in Edentulous Mandible. J Oral Biol. 2018; 5(1): 6.

Periodontology and Implant Dentistry at the New York University College of Dentistry (NYUCD) Kriser Dental Center. The ID was certified by the Office of Quality Assurance at NYUCD. This study is in compliance with the Health Insurance Portability and Accountability Act (HIPAA) requirements and approved by the University Committee on activities involving human subjects.

Thirteen consecutive cases from the database that were treated with an IL protocol utilizing the paralleling device were included in this study.

Patient inclusion criteria: All of the included patients had:

1) A total of 3-6 implants placed and immediately loaded on the same day of the surgery with a chair-side provisional restoration.

2) Totally edentulous mandible or partially edentulous mandible with more than 5 teeth that required extraction in the anterior mandible due to periodontal disease, fracture or failure of endodontic treatment.

3) An adequate anterior-posterior (A-P) spread that was present in the lower arch.

4) The treatment sites were limited to the interforamina area. A total of 9 edentulous and 5 partially edentulous mandibular cases were selected to be included in the study.

5) All cases need to be treated with same technique steps and device.

Patient exclusion criteria: All patients that were excluded from the study had:

1) Systemic diseases that could alter the tissue integration of dental implants.

2) Generalized active periodontal disease or active periapical infection in the remaining teeth.

3) Parafunctional habits.

4) Lack of compliance.

5) Bone grafts at the time of placement

Clinical procedures: A set protocol was established, standardized and applied to all 14 patients as follows;

1) Clinical and radiographic examinations were made (Figures $1-5)$.

2) Pre-surgical steps - Stereolithographic model is printed using the CBCT scan of the edentulous mandibular arch (Figure 6). Vacuum form shells and resin shells fabricated from an ideal wax up or pre-existing denture, and a bite registration on the occlusal surface of the shell with pattern resin (GC corporation, Tokyo, Japan) to evaluate the vertical dimension of occlusion.

3) Subjects took $2 \mathrm{~g}$ Amoxicillin (TEVA, USA) 1 hour prior to surgery and in those allergic to Penicillin, $600 \mathrm{mg}$ of Clindamycin (Pharmaderm, Princeton, NJ) was given.

4) Mid-crestal and when necessary vertical incisions were made after local anesthesia infiltration (2\% lidocaine 1:100,000 epinephrine [Henry Schein USA]).
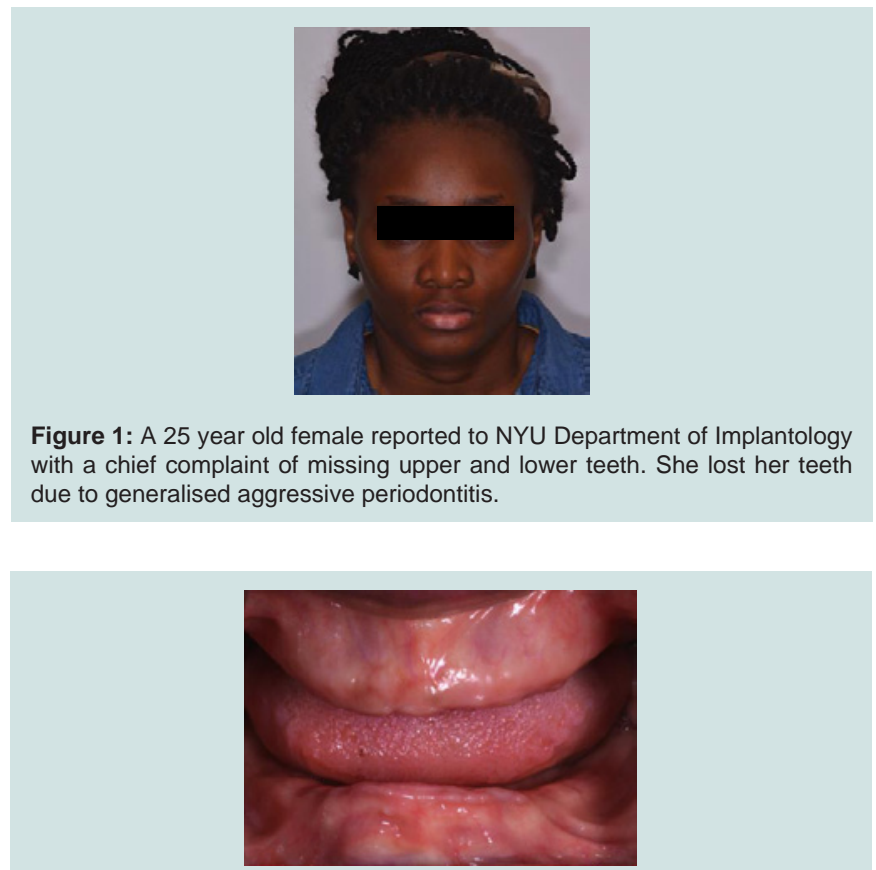

Figure 2: Completely edentulous maxillary and mandibular arches with adequate inter-ridge distance.

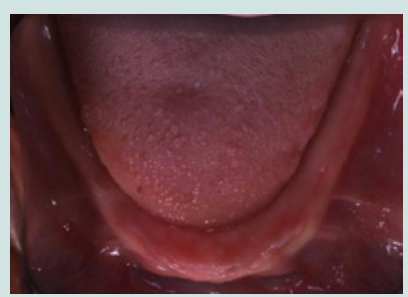

Figure 3: Edentulous mandibular ridge.

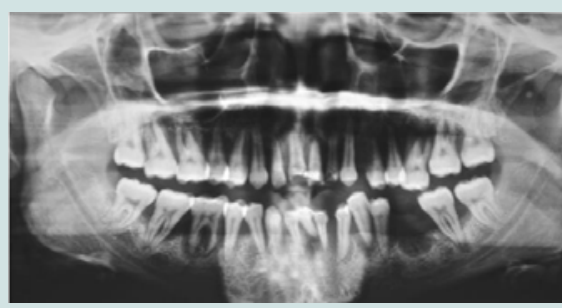

Figure 4: Panoramic radiograph shows severe bone loss around all teeth in maxillary and mandibular arches indicative of aggressive generalised periodontitis.

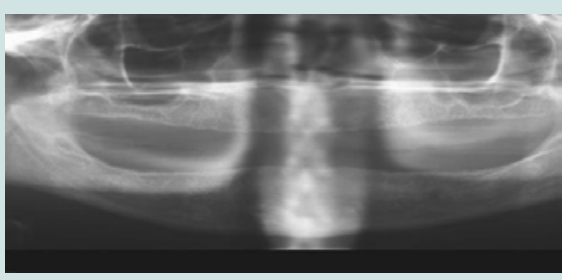

Figure 5: Panoramic radiograph shows complete loss of teeth within a span of three years. 
Citation: Kim KB, Mandali B, Wang WCW, Bagheri Z. Case Presentations and Evolution in Concepts of Immediate Loading in Edentulous Mandible. J Oral Biol. 2018; 5(1): 6.

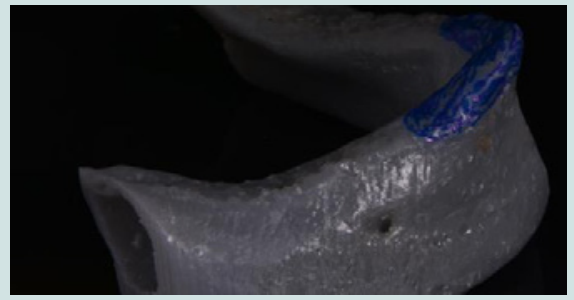

Figure 6: Stereolithographic model of the planned alveoloplasty site. The surgical paralleling guide is adjusted in accordance with the planned alveoloplasty procedure in the model prior to surgery.

5) Atraumatic tooth extraction took place in cases where immediate implant placement was performed.

6) Full thickness mucoperiosteal flaps were reflected.

7) Alveoloplasty was performed in cases where the alveolar ridge required contouring using dental bur.

8) A total of 3-6 implants were placed in each mandible using an adjustable paralleling device. The implant placement utilizing an IL protocol featuring implant surfaces with either a Resorbable Blast Media (RBM) , 4.0mm or Sandblasted, Large grit, Acid-etched (SLA) surface in the endosseous portion (Figure 7). A crestally widened implant platform diameter of $4.8 \mathrm{~mm}$, a body diameter of 3.3 or $4.1 \mathrm{~mm}$ and a length of 12 or $13 \mathrm{~mm}$ (Sybron XRT, Orange, CA, Straumann Standard Plus, Basel, Switzerland Nobel Biocare Branemark MkIII TiUnite, Balsberg, Switzerland) were used. Primary stability of each implant was achieved by undersizing the osteotomy and engaging a widened crestal platform of the implant with the cortical bone. This meant that the 2 different types of implants were placed differently. The RBM surfaced implants with the micro thread up to the platform were placed evenly with the crestal bone. The SLA surfaced implants were placed $2 \mathrm{~mm}$ more apical than the roughened surface in order to engage the widened neck with the crestal bone. Primary stability of more than $30 \mathrm{Ncm}$ was confirmed for each placement using a torque driver.

9) The narrow diameter abutments (platform switching concept) were connected at a torque of more than $30 \mathrm{Ncm}$ and wound closure was achieved using 4.0 resorbable sutures (Vicryl Ethicon).

10) A fixed provisional restoration was fabricated chair-side for cross-arch stabilization. The Omnivac shell or resin shell was mounted to verify that the abutment fit in the template. Prefabricated plastic copings were placed on the abutments and a self-curing acrylic resin or Bis-acryl Luxatemp (DMG, Hamburg, Germany) was then injected into the template (Figure 10). The template was seated with bite registration and guidance from adjacent teeth and/or the opposite jaw, and allowed to set for 4 minutes while the patient was biting in maximum intercuspation. After the temporary prosthesis was removed from the abutment, excess material was trimmed outside the mouth and occlusal adjustments were made.

11) The provisional restoration was cemented with Polycarboxylic cement (Durelon 3M ESPE, Norristown, PA) and all implants

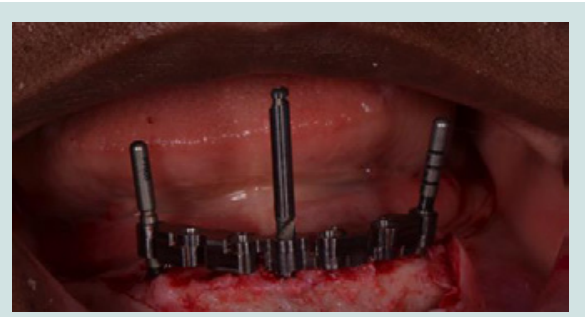

Figure 7: Paralleling Guide Block (EBI, Kyungsan, Korea) placed in the osteotomy sites confirming the parallelism of the preparation of osteotomies.

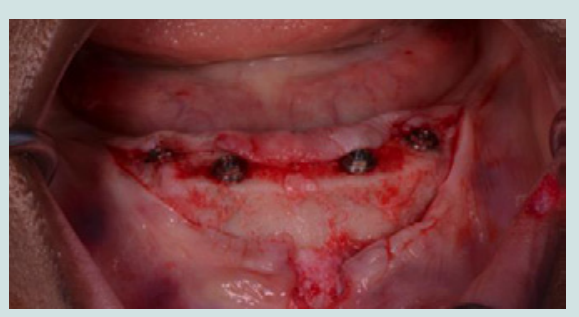

Figure 8: Implant placement after confirming their angulation and position using the guide.

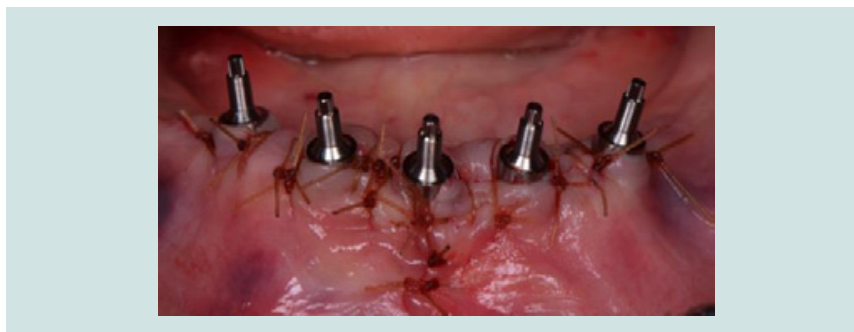

Figure 9: Post implant placement and suturing the flap, temporary abutments are connected to the implants.

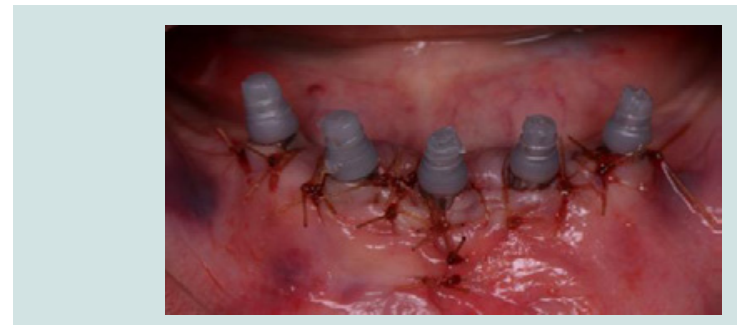

Figure 10: Temporary printed copings are placed on the abutments to receive the splinted provisional bridge.

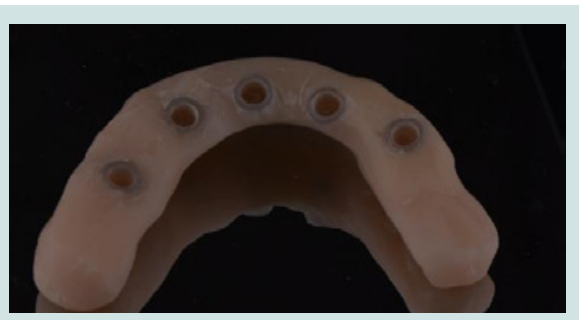

Figure 11: Immediate provisional splinted restoration prevents macro-motion to avoid disruption of osseointegration. 
Citation: Kim KB, Mandali B, Wang WCW, Bagheri Z. Case Presentations and Evolution in Concepts of Immediate Loading in Edentulous Mandible. J Oral Biol. 2018; 5(1): 6.

were immediately loaded in function (Figures 11 and 12).

12) The patient presented for postoperative care at 1 week, 2 weeks and then once every month following implant placement to monitor oral hygiene, mucosal healing, the stability of the provisional bridge, and fixture status. The patients were instructed to follow a soft diet for 6-8 weeks after surgery. Each patient was prescribed antibiotics (Amoxicillin 500 mg TID or Clindamycin $150 \mathrm{mg}$ QID) and Chlorhexidine gluconate for 1 week following the surgery (Figure 13).

13) The provisional prosthesis was not removed to avoid an excess of micro movement until the healing period was completed. Follow up panoramic radiographs were taken.

14) After 3 months of healing period, the provisional restoration was removed to evaluate implant mobility and periimplant soft tissue.

15) The definitive prosthesis was fabricated after a final impression was obtained 3-6 months after implant placement.

\section{Results}

In this case series the average survival rate was $98.4 \%$ with a loading period of 6-28 months. Out of thirteen patients, twelve demonstrated $100 \%$ implant success rate. Only one implant failed in a patient whom received 5 implants with immediate placement. One provisional restoration fractured and two cases with cement wash out during the follow-up period (Table 1).

\section{Discussion}

The immediate loading (IL) of implant-supported fixed prostheses is clinically well documented with a reported $85.7-100 \%$ implant survival rate in the edentulous mandible [15]. Immediate

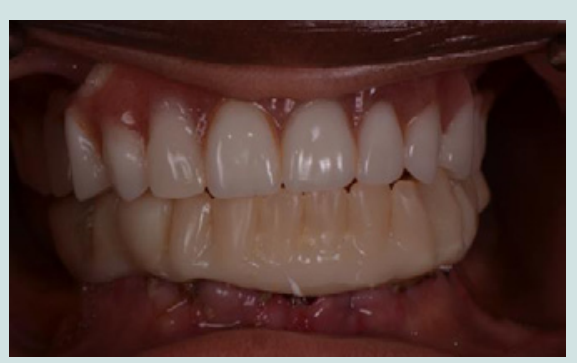

Figure 12: Post-operative photograph with immediate provisional delivered on the day of surgery.

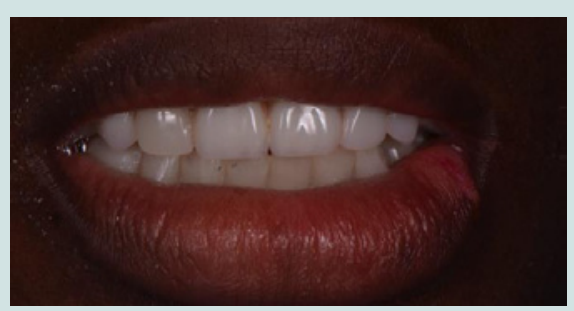

Figure 13: Post-operative photograph at 2 weeks.
Table 1: Results of the study.

\begin{tabular}{|c|c|c|c|c|c|c|c|c|}
\hline Pt & Type & $\begin{array}{c}\text { Opposite } \\
\text { Arch }\end{array}$ & Manufacturer & Implant & $\begin{array}{l}\text { Size } \\
(\mathrm{mm})\end{array}$ & $\begin{array}{l}\text { Follow } \\
\text { up } \\
\text { (mo) }\end{array}$ & $\begin{array}{l}\text { Survival } \\
\text { Rate (\%) }\end{array}$ & $\mathrm{Cx}$ \\
\hline 1 & TE & NT & Sybron & 4 & $3.3 / 4.1 \times 13$ & 28 & 100 & \\
\hline 2 & PE & FP & Sybron & 4 & $4.1 \times 13$ & 22 & 100 & \\
\hline 3 & TE & FP & Sybron & 6 & $3.3 \times 13$ & 17 & 100 & \\
\hline 4 & TE & NT/FP & Sybron & 6 & $3.3 / 4.1 \times 13$ & 16 & 100 & $\begin{array}{c}\text { Fx } \\
(4 \mathrm{mo})\end{array}$ \\
\hline 5 & TE & RP & Sybron & 5 & $4.1 \times 13$ & 15 & 100 & $\begin{array}{c}\text { CW } \\
(3 \mathrm{mo})\end{array}$ \\
\hline 6 & TE & FP & Sybron & 4 & $4.1 \times 13$ & 13 & 100 & $\begin{array}{c}\text { CW } \\
(3 \mathrm{mo})\end{array}$ \\
\hline 7 & TE & FP & Straumann & 5 & $4.1 \times 13$ & 13 & 100 & \\
\hline 8 & TE & NT & Str/Sybron & 5 & $4.1 \times 13 / 14$ & 6 & 100 & \\
\hline 9 & $\mathrm{PE}$ & $\mathrm{FP}$ & Sybron & 5 & $4.1 \times 13$ & 6 & 100 & \\
\hline 10 & $\mathrm{PE}$ & $\mathrm{FP}$ & Sybron & 4 & $4.1 \times 13$ & 6 & 100 & \\
\hline 11 & TE & FP & Sybron & 5 & $4.1 \times 13$ & 10 & 100 & \\
\hline 12 & PE & NT & Sybron & 5 & $4.1 \times 13$ & 14 & 80 & \\
\hline 13 & $\mathrm{PE}$ & NT & Sybron & 5 & $4.1 \times 13$ & 10 & 100 & \\
\hline
\end{tabular}

PE: Partial Edentulous; TE: Total Edentulous; NT: Natural Teeth; FP: Fixed Prothesis; RP: Removable Prothesis; NT/FP: Natural Teeth/Fixed prosthesis combination

loading of immediately placed implant has also shown a high success rate of $97.7-100 \%$ comparable to immediate loading of two stage implant as long as primary stability achieved $[10,16,17]$. The results of the present study indicate similar implant survival rates [18]. In this case series, a total of 63 implants were placed in 13 patients using the paralleling device and all immediately loaded. The average survival rate was $98.4 \%$ with a loading period of 6-28 months. Primary stability of more than $30 \mathrm{Ncm}$ of torque was achieved by under- sizing the osteotomy and engaging the widened crestal platform of the implant with the cortical bone. There was one implant failure. This implant was placed immediately after extraction. Authors believe the failure was due to minimum primary stability and micromotion.

One provisional restoration fractured and two presented with cement wash out during the follow-up period. However in all cases the prosthesis was maintained in place during the first 2-4 months of the healing phase.

Other critical factors for implant success are achieving optimal implant position and angulation from next page. Creating a flat bony contour is the initial step to establish optimal implant position and angulation as well as to define anatomy to maximize implant fixation for immediate load prosthesis [19]. This osteoplasty creates a flat contour on which the restoration is placed. The plat contour serves multiple functions for both the surgeon and the prosthodontist. That include:

1. Establishing prosthetic restorative space.

2. Establishing a level alveolar plane and uniform implant levels.

3. Establishing alveolar width for implant diameter selection.

4. Bone reduction makes basal bone accessible for implant fixation.

5. Helps to establish arch form, implant distribution and 
anterior posterior spread.

6. Identifies optimal implant sites.

In the mandible, segmental over eruption of the ridge and/or uneven alveolar crest are common findings. The ridge contouring bur is used to contour the ridge and establish a flat bony contour which helps implant placement at the same level and helps establish a prosthetic platform.

Optimal implant position in terms of ideal inter-implant distance can be achieved if the implant is placed in the tooth position. This can be done by using a surgical guide which is made from an ideal wax up that is prepared pre-surgically. However, implant placement also depends on the anatomy and morphology of bone and sometimes implants cannot be placed in the ideal tooth position due to lack of bone availability. Computer generated surgical guide can help optimizing the implant placement. However it requires the use of special softwares and certain expertise making the procedure costly. The technique introduced here can produce similar predictable results while being more cost effective. A pre-operative step essential to treatment planning the implant position is to obtain a $3 \mathrm{D}$ stereolithographic model of the mandible.

Surgical templates facilitate placement of implants in the planned position and can be created with planning software using computed tomography $(\mathrm{CT})$ or cone beam computed tomography (CBCT) data. Another option would be to plan and manufacture the guides on 3D stereolithographic models. Planning implant positions with computer-based methods and stereolithographic surgical templates achieves high cumulative survival rates (CSR) for implants at 1 year of follow-up [20]. In such an approach, bone reduction is first performed directly on the $3 \mathrm{D}$ model; drilling is performed by the oral surgeon. The surgeon then performs the implant osteotomies directly on the 3D models. This is followed by fabrication of both the bone reduction guide and the surgical guides. The 3D model allows further evaluation of the planned placement according to the anatomical structure of the patient prior to actual placement and manufacture of the guide on that basis. Aires I et al. conducted a retrospective study of implants placed and immediately loaded using 3D stereolithographic models [21]. Patients with failing dentition received implants and final multiunit abutments on the same day of surgery. In addition to the benefits to the patients, $3 \mathrm{D}$ modeling is viewed as being particularly user-friendly among craniofacial surgeons and highly reliable and accurate [22,23]. Three-dimensional modeling in contrast to computer planning of implant surgery does not require specific software. Furthermore, while computer planning only allows the surgeon to see the bone structure on a two-dimensional screen, 3D models enable the surgeon to physically examine the bone structure at all angles and use this information to formulate a step-by-step approach to the implant placement.

After the position of the implant has been identified, the direction/inclination of the implant must be determined. Establishing mesiodistal and buccolingual parallelism during implant placement is an important step. Adverse directions can impair the esthetics and function of the future restoration. Mesiodistal parallelism can be achieved using a surgical guide or the paralleling device. Surgical guides that are made custom based can add to the cost of the technique and their insertion require extra step. The paralleling device introduced here can be reused after sterilization, makes the technique more simple and cost effective.

It has been reported that the critical factors for successful IL are implant stability upon insertion and cross arch splinting via a rigid implant supported bridge to decrease micromotion less than 50-150 $\mathrm{nm}$ at the bone-implant interface [24]. If three or more implants are placed in a tripod or a cross-arch configuration, splinting a provisional bridge as soon as possible after implant placement reduces lateral forces on implants and allows more favorable axial forces $[3,25,26]$.

Temporization in IL can be accomplished using different techniques. These include use of an Omnivac shell to prepare chairside cement retained provisional, use of a premade resin shell to fabricate a cement-retained provisional, use of lab processed provisional or conversion of an existing denture into a screw-retained provisional [18]. Since the late 1990's, cement-retained restorations have been the preferred method with high success rates while aiding IL restorative time reduction $[19,27]$. Screw-retained restorations are more technique sensitive and time consuming when fabricated chairside. Moreover, cost is increased due to the additional laboratory and clinical components, and it is difficult to obtain non-passive bridgework [28]. Parallel alignment avoids use of angled abutment or abutment adjustment [29]. Bony stress is concentrated in the cervical area of an implant, therefore cervical cortical bone serves as the major anchoring point for an implant [3,29]. Parallel implant placement also reduces the stresses at the implant and bone interface since the implant does not have a PDL which acts as a shock absorber in teeth. A midline guide pin and a paralleling device may be used to obtain good parallelism. Other factors for successful provisionalization with an IL protocol include careful cementation. Permanent cementation is recommended because the prosthesis must remain fixed during the first 8 to 12 weeks post-surgically otherwise implant stability and osseointegration may be compromised. Additional care must be taken to limit cement extrusion beyond the abutment restoration margin and into the periimplant mucosal tissues because residual cement may cause inflammation and compromise bone and soft tissue healing [30].

\section{Conclusion}

Results of this case series demonstrated that implant placement in optimal position and angulation can be easily achieved using the suggested innovative immediate loading technique using paralleling device producing predictable results. Employing a cement retained provisional restoration with this technique is simpler, faster and more cost effective than the screw-retained protocol. Nevertheless, future prospective studies with long-term follow-up and an increased number of cases are necessary to further evaluate this technique.

\section{References}

1. Schnitman PA, Wohrle PS, Rubenstein JE (1990) Immediate fixed interim prostheses supported by two- stage threaded implants: methodology and results. J Oral Implantol 16: 96-105.

2. Balshi TJ, Wolfinger GJ (1997) Immediate loading of Brånemark implants in edentulous mandibles: a preliminary report. Implant Dent 6: 83-88.

3. Tarnow DP, Emtiaz S, Classi A (1997) Immediate loading of threaded implants at stage 1 surgery in edentulous arches: ten consecutive case reports with 
Citation: Kim KB, Mandali B, Wang WCW, Bagheri Z. Case Presentations and Evolution in Concepts of Immediate Loading in Edentulous Mandible. J Oral Biol. 2018; 5(1): 6.

1- to 5-year data. Int J Oral Maxillofac Implants 12: 319-324.

4. Horiuchi K, Uchida H, Yamamoto K, Sugimura M (2000) Immediate loading of Brånemark system implants following placement in edentulous patients: a clinical report. Int J Oral Maxillofac Implants 15: 824-830.

5. Castellon P, Block MS, Smith MB Finger IM (2004) Immediate loading of the edentulous mandible: delivery of the final restoration or a provisional restoration--which method to use? J Oral Maxillofac Surg 62 (9 Suppl 2): 30-40.

6. Buser D, Weber HP, Brägger U (1990) The treatment of partially edentulous patients with ITI hollow-screw implants: presurgical evaluation and surgical procedures. Int J Oral Maxillofac Implants 5: 165-175.

7. Ericsson I, Randow K, Glantz PO, Lindhe J, Nilner K (1994) Clinical and radiographical features of submerged and nonsubmerged titanium implants. Clin Oral Implants Res 5: 185-189.

8. Ericsson I, Randow K, Nilner K, Petersson A (1997) Some clinical and radiographical features of submerged and non-submerged titanium implants. A 5-year follow-up study. Clin Oral Implants Res 8: 422-426.

9. Grunder $U$ (2001) Immediate functional loading of immediate implants in edentulous arches: two-year results. Int J Periodontics Restorative Dent 21: 545-551.

10. Capelli M, Zuffetti F, Del Fabbro M, Testori T (2007) Immediate rehabilitation of the completely edentulous jaw with fixed prostheses supported by either upright or tilted implants: a multicenter clinical study. Int J Oral Maxillofac Implants 22: 639-644.

11. Tealdo T, Bevilacqua M, Pera F, Menini M, Ravera G, et al. (2008) Immediate function with fixed implant-supported maxillary dentures: a 12-month pilot study. J Prosthet Dent 99: 351-360.

12. Maló P, Rangert B, Nobre M (2005) All-on-4-immediate-function concept with Brånemark system implants for completely edentulous maxillae: a 1-year retrospective clinical study. Clin Implant Dent Relat Res 7 Suppl 1: S88-S94.

13. Chiapasco M, Gatti C, Rossi E, Haefliger W, Markwalder TH (1997) Implantretained mandibular overdentures with immediate loading. A retrospective multicenter study on 226 consecutive cases. Clin Oral Implant Res 8: 48-57.

14. Ostman PO (2008) Immediate/early loading of dental implants. Clinical documentation and presentation of a treatment concept. Periodontol 2000 47: 90-112.

15. Peñarrocha M, Boronat A, Garcia B (2009) Immediate loading of immediate mandibular implants with a full-arch fixed prosthesis: a preliminary study. $J$ Oral Maxillofac Surg 67: 1286-1293.

16. Szmukler-Moncler S, Salama H, Reingewirtz Y, Dubruille JH (1998) Timing of loading and effect of micro motion on bone-dental implant interface: review of experimental literature. J Biomed Mater Res 43: 192-203.

17. Romanos G, Froum S, Hery C, Cho SC, Tarnow D (2010) Survival rate of immediately vs delayed loaded implants: analysis of the current literature. J Oral Implantol 36: 315-324.

18. Misch CM (2004) Immediate loading of definitive implants in the edentulous mandible using a fixed provisional prosthesis: the denture conversion technique. J Oral Maxillofac Surg 62 (9 Suppl 2): 106-115

19. Romanos GE, Nentwig GH (2008) Immediate loading using cross-arch fixed restorations in heavy smokers: nine consecutive case reports for edentulous arches. Int J Oral Maxillofac Implants 23: 513-519.

20. Malo P, de Araujo Nobre M, Lopes A (2007) The use of computer-guided flapless implant surgery and four implants placed in immediate function to support a fixed denture: preliminary results after a mean follow-up period of thirteen months. J Prosthet Dent 97 (6 Suppl): S26-S34.

21. Aires I, Berger J (2016) Planning implant placement on 3D stereolithographic models applied with immediate loading of implant-supported hybrid prostheses after multiple extractions: a case series. Int J Oral Maxillofac Implants 31: 172-178.

22. D'Urso PS, Atkinson RL, Lanigan MW, Earwaker WJ, Bruce IJ, et al. (1998) Stereolithographic (SL) biomodelling in craniofacial surgery. Br J Plast Surg 51: 522-530.

23. Rosenfeld AL, Mandelaris GA, Tardieu PB (2006) Prosthetically directed implant placement using computer software to ensure precise placement and predictable prosthetic outcomes. Part 3: stereolithographic drilling guides that do not require bone exposure and the immediate delivery of teeth. Int J Periodontics Restorative Dent 26: 493-499.

24. Rangert B, Jemt T, Jörneus L (1989) Forces and moments on Branemark implants. Int J Oral Maxillofac Implants 4: 241-247.

25. Rangert BR, Sullivan RM, Jemt TM (1997) Load factor control for implants in the posterior partially edentulous segment. Int J Oral Maxillofac Implants 12: $360-370$.

26. Jensen OT, Adams MW, Cottam JR, Parel SM, Phillips WR $3^{\text {rd }}$ (2011) The all on 4 shelf: mandible. J Oral Maxillofac Surg 69: 175-181.

27. Bocklage R (2002) Rehabilitation of the edentulous maxilla and mandible with fixed implant-supported restorations applying immediate functional loading: a treatment concept. Implant Dent 11: 154-158.

28. Cooper L, De Kok IJ, Reside GJ, Pungpapong P, Rojas-Vizcaya F (2005) Immediate fixed restoration of the edentulous maxilla after implant placement. J Oral Maxillofac Surg 63 (9 Suppl 2): 97-110.

29. Pierrisnard L, Renouard F, Renault $P$, Barquins $M$ (2003) Influence of implant length and bicortical anchorage on implant stress distribution. Clin Implant Dent Relat Res 5: 254-262.

30. Pauletto N, Lahiffe BJ, Walton JN (1999) Complications associated with excess cement around crowns on osseointegrated implants: a clinical report. Int J Oral Maxillofac Implants 14: 865-868. 